\title{
Hormones and Cancer Reviewers
}

(C) Springer Science+Business Media, LLC 2010

We wish to thank the following reviewers for their time and effort on behalf of Hormones and Cancer:

Kimberly Allison

Yoland Antill

Michael C. Archer

Rolf Buslei

Maria V. Barbolina

Diana Benn

Patricia Berg

Alexander D. Borowsky

Nathan J. Bowen

William Brinkley

Angela Brodie

Sally Camper

Tobias Carling

Vincent Cavaillès

Subhankar Chakraborty

Wendy Y. Chen

Sheue-Yann Cheng

Daniela Cocchi

Ronny Drapkin

Karam El-Bayoumy

Maria Grazia Chiofalo

Marc Gunter

Susan Hankinson

Kate Horwitz

Nuhad K. Ibrahim
Twila Jackson

Virgil Craig Jordan

Twila Jackson

Virginia Kaklamani

Benita Katzenellenbogen

Larry Kirschner

Carolyn M. Klinge

Srinivas Koduru

Carol Lange

Alexander Lazar

Adrian V. Lee

Christopher I. Li

Jonathan J. Li

Ming-Fong Lin

Wilma Lingle

Michaela Luconi

Annekatrin Lukanova

M. Mannelli

Constantine S. Mitsiades

James L. Mohler

Peter Nelson

Simon Newman Newman

John J. Peluso

Michael Pollak

Laszlo G. Radvanyi
Lois M. Ramondetta

Hein te Riele

Pradip Roy-Burman

Henri Rochefort

Michel M. Sanders

Deepa Sathyaseelan

Victoria Seewaldt

Subrata Sen

Jeremy A. Squire

Anil Sood

Maamar Souidi

Susan Sturgeon

Ossama W. Tawfik

Wayne Tilley

Donald James Tindall

Amy Trentham-Dietz

Marcel (ThB) Twickler

Hsien-yu Wang

Nancy L. Weigel

Alessandro Weisz

Dan Welch

Thilo Welsch

Regina G. Ziegler

Anne Zeleniuch-Jacquotte

Douglas Yee 